\title{
Characterization of a magnetic nanoparticle manipulation system. Towards HUS diagnosis.
}

\author{
Claudio Ferrari ${ }^{1,2}$, Ariel Kukulanski ${ }^{1,2}$, Marcelo Vasquez Mansilla ${ }^{2,4,5}$, Enio Lima Junior $^{2,4,5}$, Salvador \\ Ortiz ${ }^{1,2}$, Roberto Zysler $^{2,4,5}$, Guido Berlin $^{1,2}$, Laura Steren ${ }^{1,3,5,6}$, Cintia Notcovich $^{1,2}$ \\ ${ }^{1}$ Micro and Nanotechnology Department, CNEA, CAC, Argentina \\ 2 Institute of Nanoscience and Nanotechnology, Argentina \\ ${ }^{3}$ Laboratory of Magnetic nanostructures and devices, CNEA, CAC, Argentina \\ ${ }^{4}$ Laboratory of Magnetic Resonance, CNEA, CAB Argentina \\ ${ }^{5}$ CONICET, Argentina \\ ${ }^{6}$ FCEN, UBA, Argentina
}

Corresponding author: notcovich@cnea.gov.ar

\begin{abstract}
In the context of the development of a magnetic sensor with the goal of early detection of HUS, we develop a system for manipulation of Superparamagnetic nanoparticles. We report some preliminary theoretical and experimental results.
\end{abstract}

Key words: Magnetic Biosensors, magnetic nanoparticles, HUS.

\section{Introduction}

The Hemolytic Uremic Syndrome (HUS) is the main cause of acute renal failure and the second cause of chronic renal failure and renal transplant in Argentina. Our country has the highest HUS registry in the world $[1,2]$. It is a disease caused by a bacterium that produces a toxin, the Shiga toxin. The bacterium is transmitted to humans mainly through contaminated food, such as products of undercooked meat and raw milk.

This work is part of a project aimed at developing a biosensor capable of providing an early diagnosis of HUS. In recent years, phenomena discovered in artificial nanostructures such as giant magnetoresistance (GMR) have led to the development of new devices for the detection of biomolecules. In these new elements, nano or micro magnetic particles are used as tags of biomolecules that are detected with magnetic field sensors [3].

An important advantage of "magnetic labels" over fluorescents, for example, is the low sample volume required. In addition, magnetoresistive sensors, among other advantages, provide the device with high sensitivity, rapid response and the possibility of being integrated into multi-analysis detection lab-on-chip devices, reducing time and cost of the tests. The detailed knowledge of the behavior of the magnetic particles within the microfluidic circuit of the device is fundamental for the design of the geometry of the sensor and other components of the lab-on-chip dedicated to controlling the dynamics of the particles.

\section{Dynamics of Magnetic Microparticles.}

One of the key aspects of building a functioning magnetic sensor is the manipulation of magnetic particles. With this in mind, we proposed in a first stage to synthesize magnetic microparticles and characterize their dynamics. In a PDMS / glass microchannel, $\mathrm{Fe}_{3} \mathrm{O}_{4}$ microparticles (magnetite) obtained by mechanical grinding were used. These particles (Size varying between 1 and $3 \mu \mathrm{m}$ ), were characterized. We studied the influence of different parameters of interest such as the magnetic moment of each particle or the drag force of the fluid. The magnetic properties of the particles were examined with magnetization measurements as a function of magnetic field and temperature.

The dynamics of the particles immersed in an aqueous medium and subjected to a magnetic field inside a microfluidic channel was 
evaluated by optical microscopy. The field was provided by an external magnet.

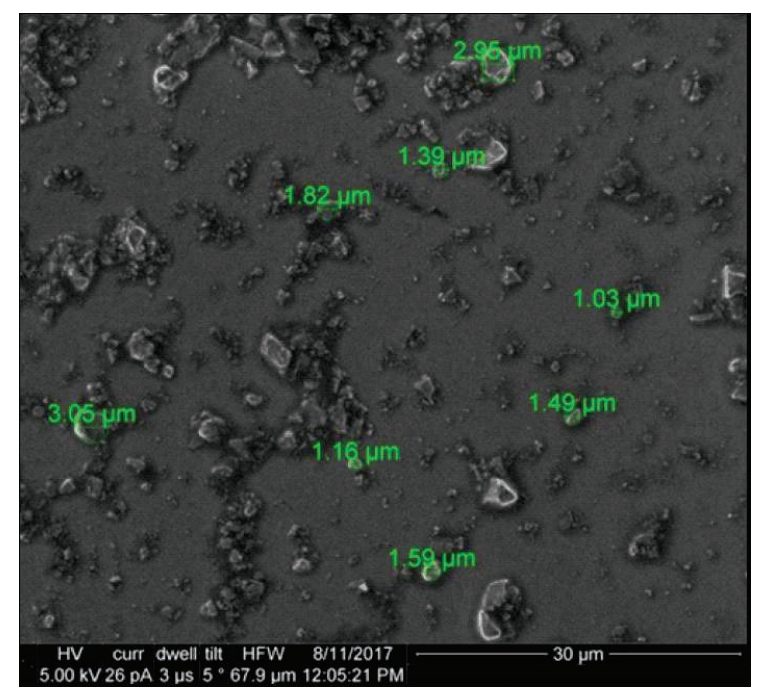

Fig. 1. SEM capture of the particles.

This allowed us to verify that the particles reach the limit velocity within a negligible time interval, and then travel with constant speed. It was possible to correlate the particle size with its mobility, as shown in Fig. 2.

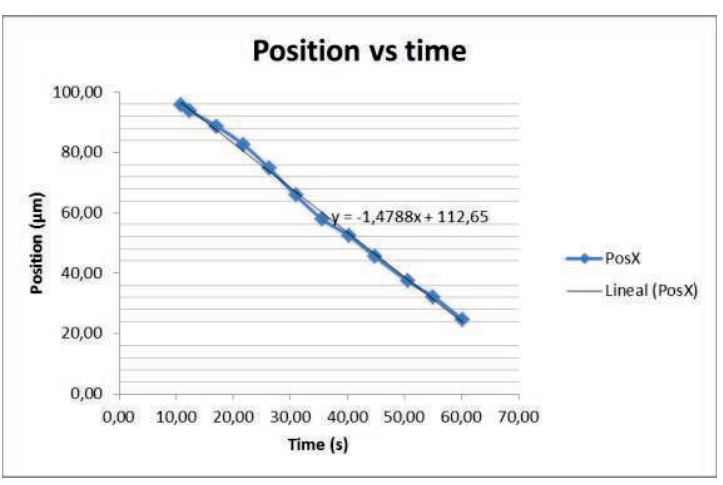

Fig. 2. Position vs time for a magnetic particle. The limit velocity is reached at the beginning of the dynamical evolution.

\section{Generation of a magnetic field for particle manipulation on a chip}

The next step in our road towards an integrated magnetic sensor is to generate and control the magnetic field on board the chip. For this purpose, we explored different alternatives, and settled on a system that consists of a few strips of Au deposited on a Si wafer. Our first step was to simulate the behavior of the magnetic field with this geometry. We considered a substrate of Si and water covering the whole setup, to better mimic the real operational conditions of the sensor. After calculating the magnetic field, using the distribution of field lines, we were able to determine the force considering in principle that the magnetization of the particles is saturated. The expression for the force reads [4]

$\vec{F}_{m}=\frac{V_{m a g} \Delta \chi}{\mu_{0}}(\vec{B} \cdot \vec{\nabla}) \vec{B}(1)$,

where $V_{\text {mag }}$ is the volume of the particle, $\Delta \chi$ is the difference in volume susceptibility between the particle and the medium that surrounds it and $\mu_{0}$ is the vacuum permeability. The force around one of the strips was plotted and shown in Fig.3.

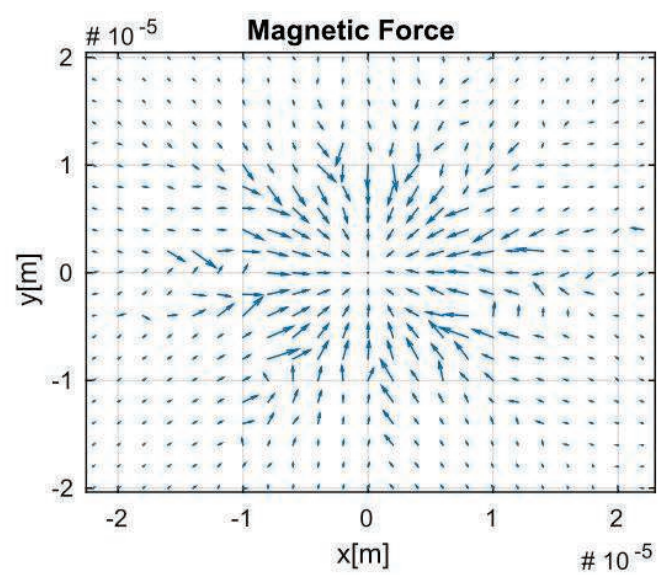

Fig. 3. Magnetic force around a conducting strip

This shows us that alternately turning on and off the current strips allows us to concentrate the particles and transport them in this fashion towards the sensing region.

Considering the theoretical results we built an experimental set-up consisting on a series of stripes deposited on a Si wafer, and a Polydimethylsiloxane (PDMS) enclosure. The PDMS and $\mathrm{Si}$ surfaces were activated by plasma and put in contact in order to bind them irreversibly. We filled this volume with water. This allowed us to study the dynamics of the magnetic nanoparticles in real conditions.

\section{References}

[1] H. Karch, P.I. Tarr, M. Bielaszewska, Enterohaemorrhagic Escherichia coli in human medicine. Int. J. Med. Microbiol. 295, 405-418 (2005).

[2] H. A. Repetto, Long-term course and mechanisms of progression of renal disease inhemolytic uremic syndrome. Kidney Int. S102S106 (2005)

[3] D. A. Halla, et al. GMR Biosensor Arrays: A System Perspective. Biosens Bioelectron. 2010 May 15; 25(9): 2051-2057.

[4] Gooneratne C. P et al, On-Chip Magnetic Bead Manipulation and Detection Using a Magnetoresistive Sensor-Based Micro-Chip: Design Considerations and Experimental Characterization. Sensors, 2016, 16, 1369; doi:10.3390/s16091369 